Z. Wahrscheinlichkeitstheorie verw. Geb. 8, 12-18 (1967)

\title{
On the Influence of Moments on the Rate of Convergence to the Normal Distribution
}

\author{
C. C. Heyde
}

Received July 26, 1966 


\section{Introduction}

Let $X_{i}, i=1,2,3, \ldots$ be a sequence of independent and identically distributed random variables with $\operatorname{var} X_{i}=\sigma^{2}<\infty$ and $E X_{i}=0$. Write

Then,

$$
S_{n}=\sum_{i=1}^{n} X_{i}, \quad n \geqq 1
$$

$$
F_{n}(x)=\operatorname{Pr}\left(S_{n} \leqq \sigma \sqrt{n} x\right) \rightarrow \Phi(x)=\frac{1}{\sqrt{2 \pi}} \int_{-\infty}^{x} e^{-\frac{1}{2} u^{2}} d u
$$

as $n \rightarrow \infty$, and the rate of this convergence is a matter of considerable importance. In this paper we shall establish the following theorem:

Theorem. Let $X_{i}, i=1,2,3, \ldots$ be a sequence of independent and identically distributed random variables with $E X_{i}^{2}=\sigma^{2}<\infty, E X_{i}=0$. Then,

$$
\sum_{n=1}^{\infty} n^{-1+\delta / 2} \sup _{x}\left|F_{n}(x)-\Phi(x)\right|<\infty, \quad 0 \leqq \delta<1,
$$

if and only if $E\left|X_{i}\right|^{2+\delta}<\infty, 0<\delta<1, E X_{i}^{2} \log \left(1+\left|X_{i}\right|\right)<\infty, \delta=0$.

With the exception of the boundary case $\delta=0$, this result provides a global generalization of a theorem of BAUM and KATz [1] in which they established the convergence of the series $\sum_{1}^{\infty} n^{-1+\delta / 2}\left|\operatorname{Pr}\left(S_{n} \leqq 0\right)-\frac{1}{2}\right|$ under the condition

$$
E\left|X_{i}\right|^{2+\delta}<\infty, \quad 0 \leqq \delta<1 .
$$

The theorem of this paper is, in a sense, complementary to Theorem 3.4.1 of LinNiK and Ibragrmov [7] in which they show that

if and only if

$$
\left|F_{n}(x)-\Phi(x)\right|=O\left(n^{-\delta / 2}\right), \quad 0<\delta<1,
$$

$$
\int_{|x|>z} x^{2} d F(x)=O\left(z^{-\delta}\right),
$$

where $F(x)=\operatorname{Pr}(X \leqq x)$ (a sketch of the proof of this result has also appeared in Ibragimov [5]). The large literature on the Berry-Esseen estimate is also closely related. See for example KATz [6] and BIKJALIs [2] for recent contributions in this field. A comparison of the forms of the various results would seem to indicate that moment conditions are equivalent to series conditions on the terms $\sup _{x}\left|F_{n}(x)-\Phi(x)\right|$ rather than conditions on the individual elements. 


\section{Details}

In view of our assumption of a finite variance, we can express the characteristic function $f(t)$ of the $X_{i}$ in the form

$$
f(t)=\exp \left\{-\frac{\sigma^{2} t^{2}}{2}(1+\gamma(t))\right\}
$$

where $\lim _{t \rightarrow 0} \gamma(t)=0$. Furthermore, in a neighbourhood of the origin, the equation

$$
f(t)=\exp \left\{-\frac{\sigma^{2} t^{2}}{2}\right\}
$$

can be satisfied at not more than a finite number of points ([7], §6, Chapter 1) and hence in some suitably small interval, say $0<t<\varepsilon$, we have $\gamma(t) \neq 0$.

Firstly, we shall prove the theorem for symmetric random variables $X_{i}$. In this case $f(t)$ and $\gamma(t)$ are real valued and symmetric. The proof is accomplished with the aid of two lemmas.

Lemma 1. For symmetric random variables the series (1) converges if and only if for $A>0$,

$$
\int_{0}^{A} \frac{|\gamma(u)|}{u^{1+\delta}} d u<\infty .
$$

Proof. Firstly we prove necessity. Let $f_{n}(t)$ be the characteristic function corresponding to $F_{n}(x)$. Then, integrating by parts in the equation

we obtain

$$
f_{n}(t)-e^{-t^{2} / 2}=\int_{-\infty}^{\infty} e^{i t x} d\left[F_{n}(x)-\Phi(x)\right]
$$

$$
-\frac{f_{n}(t)-e^{-t^{2} / 2}}{i t}=\int_{-\infty}^{\infty} e^{i t x}\left[\boldsymbol{F}_{n}(x)-\Phi(x)\right] d x .
$$

Further, it is seen that

$$
i t e^{-t^{2} / 2}=\int_{-\infty}^{\infty} e^{i t x} \frac{1}{\sqrt{2 \pi}} x e^{-x^{2} / 2} d x .
$$

We observe that the conditions for the applicability of the Parseval identity are satisfied and hence

$$
\int_{-\infty}^{\infty}\left[f_{n}(t)-e^{-t^{2} / 2}\right] e^{-t^{2} / 2} d t=\frac{1}{\sqrt{2 \pi}} \int_{-\infty}^{\infty}\left[F_{n}(x)-\Phi(x)\right] x e^{-x^{2} / 2} d x .
$$

Thus from (1)

$$
\begin{aligned}
\sum_{1}^{\infty} n^{-1+\delta / 2}\left|\int_{-\infty}^{\infty}\left[f_{n}(t)-e^{-t^{2} / 2}\right] e^{-t^{2} / 2} d t\right| & =\sum_{1}^{\infty} n^{-1+\delta / 2}\left|\int_{-\infty}^{\infty}\left[F_{n}(x)-\Phi(x)\right] x e^{-x^{2} / 2} d x\right| \\
& \leqq \sum_{1}^{\infty} n^{-1+\delta / 2} \sup _{x}\left|F_{n}(x)-\Phi(x)\right|<\infty
\end{aligned}
$$

That is,

$$
\sum_{1}^{\infty} n^{-1+\frac{1}{2} \delta}\left|\int_{-\infty}^{\infty} e^{-t^{2}}\left(1-e^{-\frac{1}{2} t^{2} \gamma\left(\frac{t}{\sigma \sqrt{n}}\right)}\right) d t\right|<\infty,
$$

and since $\gamma(u)$ does not change sign in some interval $[0, \varepsilon]$, we must have

$$
\sum_{1}^{\infty} n^{-1+\frac{1}{2} \delta} \int_{0}^{1} e^{-t^{2}}\left|1-e^{-\frac{1}{2} t^{2} \gamma(t /(\sigma \sqrt{n}))}\right| d t<\infty
$$


However, as $n \rightarrow \infty$,

$$
\left|1-e^{-\frac{1}{2} t^{2} \gamma(t /(\sigma \sqrt{n}))}\right|=\frac{1}{2} t^{2}\left|\gamma\left(\frac{t}{\sigma \sqrt{n}}\right)\right|(1+o(1)),
$$

so that

$$
\sum_{1}^{\infty} n^{-1+\frac{1}{2} \delta} \int_{0}^{1} t^{2}\left|\gamma\left(\frac{t}{\sigma \sqrt{n}}\right)\right| d t<\infty
$$

and, upon making the transformation $u=t / \sigma \sqrt{n}$, this yields

$$
\sum_{1}^{\infty} n^{\frac{1}{2}(1+\delta)} \int_{0}^{1 /(\sigma \sqrt{n})} u^{2}|\gamma(u)| d u<\infty \text {. }
$$

Now $\int_{0}^{1 /(\sigma \sqrt{x})} u^{2}|\gamma(u)| d u$ is montone decreasing as $x$ increases so, for $X>1$,

$$
\begin{aligned}
\int_{1}^{X} x^{\frac{1}{2}(1+\delta)}\left\{\int_{0}^{1 /(\sigma \sqrt{x})} u^{2}|\gamma(u)| d u\right\} d x & \leqq \sum_{n=1}^{[X]} \int_{n}^{n+1} x^{\frac{1}{2}(1+\delta)}\left\{\int_{0}^{1 /(\sigma \sqrt{x})} u^{2}|\gamma(u)| d u\right\} d x \\
& \leqq \sum_{n=1}^{[X]}(n+1)^{\frac{1}{2}(1+\delta)} \int_{0}^{1 /(\sigma \sqrt{n})} u^{2}|\gamma(u)| d u
\end{aligned}
$$

where $[X]$ is the largest integer less than or equal to $X$. Furthermore, it is possible to choose a constant $c>0$ such that $(n+1)^{\frac{1}{2}(1+\delta)}<c n^{\frac{1}{2}(1+\delta)}$ for all positive integral $n$ and hence, for all $X>1$,

$$
\int_{1}^{X} x^{\frac{1}{2}(1+\delta)}\left\{\int_{0}^{1 /(\sigma \sqrt{x})} u^{2}|\gamma(u)| d u\right\} d x<c \sum_{n=1}^{[X]} n^{\frac{1}{2}(1+\delta)} \int_{0}^{1 /(\sigma \sqrt{n})} u^{2}|\gamma(u)| d u
$$

Consequently, using (4) we see that

$$
\int_{1}^{\infty} x^{\frac{1}{2}(1+\delta)}\left\{\int_{0}^{1 /(\sigma \sqrt{x})} u^{2}|\gamma(u)| d u\right\} d x<\infty
$$

Now, in view of (5) we must have

$$
\int_{w}^{2 w} x^{\frac{1}{2}(1+\delta)}\left\{\int_{0}^{1 /(\sigma \sqrt{x})} u^{2}|\gamma(u)| d u\right\} d x \rightarrow 0 \quad \text { as } \quad w \rightarrow \infty,
$$

and

$$
\int_{w}^{2 w} x^{\frac{1}{2}(1+\delta)}\left\{\int_{0}^{1 /(\sigma / \bar{x})} u^{2}|\gamma(u)| d u\right\} d x \geqq v^{\frac{1}{2}(3+\delta)} \int_{0}^{1 /(\sigma \sqrt{2 w})} u^{2}|\gamma(u)| d u \geqq 0,
$$

so that, putting $v=1 / \sigma \sqrt{2 w}$, we conclude that

$$
\frac{1}{v^{3+\delta}} \int_{0}^{v} u^{2}|\gamma(u)| d u \rightarrow 0 \text { as } v \rightarrow 0
$$

Then, upon making the transformation $v=1 / \sigma \sqrt{x}$, (5) becomes

$$
\int_{0}^{\sigma^{-1}}\left\{\int_{0}^{v} u^{2}|\gamma(u)| d u\right\} \frac{d v}{v^{4+\delta}}<\infty
$$


and in view of $(6)$, the fact that condition (3) is satisfied follows immediately from an integration by parts.

It remains to prove the sufficiency of the condition (3). Making use of Theorem 1.5.2 of [7] we have for $T>0$,

$$
\left|F_{n}(x)-\Phi(x)\right| \leqq \frac{1}{\pi} \int_{-T}^{T}\left|\frac{f_{n}(t)-e^{-t^{2} / 2}}{t}\right| d t+\frac{24}{\pi \sqrt{2 \pi} T} .
$$

Take $T=B \sigma \sqrt{n}$ where $2 \sigma^{-1}>B>0$ is chosen so small that

$$
\max _{0 \leqq t \leqq B}|\gamma(t)| \leqq \frac{1}{2}
$$

Then, using the inequality

we have

$$
\left|e^{x}-1\right| \leqq|x| e|x|
$$

$$
\begin{aligned}
\left|f_{n}(t)-e^{-\frac{1}{2} t^{2}}\right| & =e^{-\frac{1}{2} t^{2}}\left|1-e^{-\frac{1}{2} t^{2} \gamma(t /(\sigma \sqrt{n}))}\right| \\
& \leqq \frac{1}{2} t^{2}\left|\gamma\left(\frac{t}{\sigma \sqrt{n}}\right)\right| \exp \left\{-\frac{t^{2}}{2}+\frac{t^{2}}{2}\left|\gamma\left(\frac{t}{\sigma l^{-}}\right)\right|\right\} .
\end{aligned}
$$

Hence for $|t| \leqq T$, using (8),

so that we have

$$
\left|f_{n}(t)-e^{-\frac{1}{2} t^{2}}\right| \leqq \frac{t^{2}}{2}\left|\gamma\left(\frac{t}{\sigma \sqrt{n}}\right)\right| e^{-\frac{1}{4} t^{2}}
$$

$$
\begin{aligned}
& \sum_{1}^{\infty} n^{-1+\frac{1}{2} \delta} \int_{-T}^{T}\left|\frac{f_{n}(t)-e^{-\frac{1}{2} t^{2}}}{t}\right| d t \leqq \sum_{1}^{\infty} n^{-1+\frac{1}{2} \delta} \int_{0}^{B \sigma \sqrt{n}} t\left|\gamma\left(\frac{t}{\sigma \sqrt{n}}\right)\right| e^{-\frac{1}{4} t^{2}} d t \\
= & \sigma^{2} \sum_{1}^{\infty} n^{\frac{1}{2} \delta} \int_{0}^{B} u|\gamma(u)| e^{-\frac{1}{4} \sigma^{2} n u^{2}} d u=\sigma^{2} \int_{0}^{B} u|\gamma(u)|\left\{\sum_{1}^{\infty} n^{\frac{1}{2} \delta} e^{-\frac{1}{2} \sigma^{2} n u^{2}}\right\} d u .
\end{aligned}
$$

Now, making use of a standard Abelian theorem (e.g. Feller [3], 423) we see that

$$
\lim _{S \uparrow 1}(1-S)^{1+\frac{1}{2} \delta} \sum_{n=1}^{\infty} n^{\frac{1}{2} \delta} S^{n}=\Gamma\left(1+\frac{1}{2} \delta\right),
$$

and hence, for $u \neq 0$, it is possible to choose a constant $K>0$ such that

$$
\sum_{n=1}^{\infty} n^{\frac{1}{2} \delta} e^{-\frac{1}{4} \sigma^{2} n u^{2}} \leqq K\left(1-e^{-\frac{1}{4} \sigma^{2} u^{2}}\right)^{-\left(1+\frac{1}{2} \delta\right)} .
$$

Furthermore, for $0 \leqq u \leqq B$ we have $\frac{1}{4} \sigma^{2} u^{2}<1$, so that

and hence

$$
1-e^{-\frac{1}{4} \sigma^{2} u^{2}}>\frac{1}{4} \sigma^{2} u^{2}\left(1-\frac{1}{8} \sigma^{2} u^{2}\right),
$$

$$
\sum_{n=1}^{\infty} n^{\frac{1}{2} \delta} e^{-\frac{1}{4} \sigma^{2} n u^{2}}<\frac{K 2^{2+\delta}}{\sigma^{2+\delta} u^{2+\delta}\left(1-\frac{1}{8} \sigma^{2} u^{2}\right)^{1+\frac{1}{2} \delta}} .
$$

Then, using (9) and (10) we see that

$$
\sum_{n=1}^{\infty} n^{-1+\frac{1}{2} \delta} \int_{-T}^{T}\left|\frac{f_{n}(t)-e^{-\frac{1}{2} t^{2}}}{t}\right| d t \leqq \frac{K 2^{2+\delta}}{\sigma^{\delta}} \int_{0}^{B} \frac{|\gamma(u)|}{u^{1+\delta}\left(1-\frac{1}{8} \sigma^{2} u^{2}\right)^{1+\frac{1}{2} \delta}} d u
$$


and this is finite in view of the condition (3). It follows from (7) that the condition (1) must be satisfied. This completes the proof of the lemma.

Lemma 2. For symmetric random variables, the condition (3) is satisfied if and only if $E\left|X_{i}\right|^{2+\delta}<\infty, 0<\delta<1, E X_{i}^{2} \log \left(1+\left|X_{i}\right|\right)<\infty, \delta=0$.

Proof. In a neighbourhood of the origin,

and then,

$$
f(t)-1=-\frac{\sigma^{2} t^{2}}{2}(1+o(1))
$$

$$
\log f(t)=\log \{1+[f(t)-1]\}=[f(t)-1]+O\left(t^{4}\right) .
$$

Suppose, for the sake of definiteness, that in the interval $(0, C], \gamma(t)<0$; we obtain from (11),

$$
\begin{aligned}
\frac{1}{2} \sigma^{2} \int_{0}^{C} \frac{|\gamma(t)|}{t^{1+\delta}} d t & =\int_{0}^{C} \frac{\frac{1}{2} \sigma^{2} t^{2}+\log f(t)}{t^{3+\delta}} d t=\int_{0}^{C} \frac{\frac{1}{2} \sigma^{2} t^{2}+[f(t)-1]}{t^{3+\delta}} d t+O\left(C^{2-\delta}\right) \\
& =\int_{0}^{C}\left\{\frac{\frac{1}{2} \sigma^{2} t^{2}+\int_{-\infty}^{\infty}(\cos t u-1) d F(u)}{t^{3+\delta}}\right\} d t+O\left(C^{2-\delta}\right) \\
& =\int_{-\infty}^{\infty}\left\{\int_{0}^{C}\left(\frac{\cos t u-1+\frac{1}{2} t^{2} u^{2}}{t^{3+\delta}}\right) d t\right\} d F(u)+O\left(C^{2-\delta}\right) .
\end{aligned}
$$

Consequently, the condition (3) and the condition

$$
\int_{-\infty}^{\infty}\left\{\int_{0}^{C}\left(\frac{\cos t u-1+\frac{1}{2} t^{2} u^{2}}{t^{3+\delta}}\right) d t\right\} d F(u)<\infty
$$

are equivalent.
Now let us look at the integrand $\int_{0}^{C}\left(\frac{\cos t u-1+\frac{1}{2} t^{2} u^{2}}{t^{3+\delta}}\right) d t$. After two integra-
tions by parts we obtain tions by parts we obtain

$$
\begin{aligned}
\int_{0}^{C}\left(\frac{\cos t u-1+\frac{1}{2} t^{2} u^{2}}{t^{3+\delta}}\right) d t= & -\frac{\cos C u-1+\frac{1}{2} C^{2} u^{2}}{(2+\delta) C^{2+\delta}}-\frac{u(C u-\sin C u)}{(1+\delta)(2+\delta) C^{1+\delta}}+ \\
& +\frac{u^{2}}{(2+\delta)(1+\delta)} \int_{0}^{C} \frac{1-\cos t u}{t^{1+\delta}} d t
\end{aligned}
$$

so that the condition (12) is equivalent to the condition

$$
\int_{-\infty}^{\infty} u^{2}\left\{\int_{0}^{C} \frac{1-\cos t u}{t^{1+\delta}} d t\right\} d F(u)<\infty
$$

which transforms to give

$$
\int_{-\infty}^{\infty}|u|^{2+\delta}\left\{\int_{0}^{C|u|} \frac{1-\cos v}{v^{1+\delta}} d v\right\} d F(u)<\infty
$$

(13) is obviously equivalent to the condition $E\left|X_{i}\right|^{2+\delta}<\infty$ when $0<\delta<1$. 
On the other hand, when $\delta=0$, we have for $|u|>1$

$$
\begin{aligned}
\int_{0}^{C|u|} \frac{1-\cos v}{v} d v & =\int_{0}^{C} \frac{1-\cos v}{v} d v+\int_{C}^{C|u|} \frac{d v}{v}-\int_{C}^{C|u|} \frac{\cos v}{v} d v \\
& \sim \log |u| \text { as }|u| \rightarrow \infty,
\end{aligned}
$$

so that (13) is equivalent to the condition $E X_{i}^{2} \log \left(1+\left|X_{i}\right|\right)<\infty$. This completes the proof of the lemma.

Lemmas 1 and 2 establish the theorem for the case of symmetric random variables. We go on to complete the proof of the theorem in the general case.

Firstly, we shall examine the necessity part. Consider the sequence $Y_{i}$, $i=1,2,3, \ldots$ of independent symmetrized random variables; each $Y_{i}$ having the distribution of the difference between two independent $X_{i}^{\prime}$ s. Obviously, the characteristic function of the random variables $Y_{i}$ is $|f(t)|^{2}$ and the distribution function of the sum

$$
Z_{n}=\frac{Y_{1}+\cdots+Y_{n}}{\sigma \sqrt{2 n}}
$$

is equal to $F_{n}(x \sqrt{2}) *\left(1-F_{n}(-x \sqrt{2}-0)\right)=G_{n}(x)$. Hence, if $F_{n}(x)$ satisfies the condition (1), then

$$
\begin{aligned}
& \quad \sum_{1}^{\infty} n^{-1+\frac{1}{2} \delta} \sup _{x}\left|G_{n}(x)-\Phi(x)\right| \\
& =\sum_{1}^{\infty} n^{-1+\frac{1}{2} \delta} \sup _{x}\left|F_{n}(x \sqrt{2}) *\left(1-F_{n}(-x \sqrt{2}-0)\right)-\Phi(x \sqrt{2}) *(1-\Phi(-x \sqrt{2}))\right| \\
& \leqq \sum_{1}^{\infty} n^{-1+\frac{1}{2} \delta} \sup _{x}\left|F_{n}(x \sqrt{2}) *\left(1-F_{n}(-x \sqrt{2}-0)\right)-\Phi(x \sqrt{2}) *\left(1-F_{n}(-x \sqrt{2}-0)\right)\right| \\
& +\sum_{1}^{\infty} n^{-1+\frac{1}{2} \delta} \sup _{x}\left|\Phi(x \sqrt{2}) *\left(1-F_{n}(-x \sqrt{2}-0)\right)-\Phi(x \sqrt{2}) *(1-\Phi(-x \sqrt{2}))\right| \\
& <\infty .
\end{aligned}
$$

By Lemma 1 in such a case,

$$
\int_{0}^{A} \frac{|\operatorname{Re} \gamma(t)|}{t^{1+\delta}} d t<\infty,
$$

and proceeding exactly as with the proof of Lemma 2 we extract the information that $E\left|Y_{i}\right|^{2+\delta}<\infty, 0<\delta<1, E Y_{i}^{2} \log \left(1+\left|Y_{i}\right|\right)<\infty, \delta=0$ and hence $E\left|X_{i}\right|^{2+\delta}<\infty, 0<\delta<1, E X_{i}^{2} \log \left(1+\left|X_{i}\right|\right)<\infty, \delta=0$.

In order to prove the sufficiency part, we shall make use of the result that if

$$
\int_{0}^{A} \frac{|\gamma(t)|}{t^{1+\delta}} d t<\infty, \quad 0 \leqq \delta<1,
$$

then

$$
\sum_{1}^{\infty} n^{-1+\frac{1}{2} \delta} \sup _{x}\left|F_{n}(x)-\Phi(x)\right|<\infty .
$$

We note that $|\gamma(t)|=|\gamma(-t)|$. Then, the proof of this result is essentially the same as the proof of sufficiency in Lemma 1 and is hence omitted. We complete 
the proof by showing that the condition

$$
E\left|X_{i}\right|^{2+\delta}<\infty, \quad 0<\delta<1, \quad E X_{i}^{2} \log \left(1+\left|X_{i}\right|\right)<\infty, \quad \delta=0,
$$

implies the condition (14).

Now exactly as with the proof of Lemma 2 we can show that $E\left|X_{i}\right|^{2+\delta}<\infty$, $0<\delta<1, E X_{i}^{2} \log \left(1+\left|X_{i}\right|\right)<\infty, \delta=0$ implies

$$
\int_{0}^{A} \frac{|\operatorname{Re} \gamma(t)|}{t^{1+\delta}} d t<\infty
$$

we shall show that the corresponding integral obtained by replacing $\operatorname{Re} \gamma(t)$ by $\operatorname{Im} \gamma(t)$ is also finite. Indeed, in a neighbourhood of the origin,

so that

$$
\begin{aligned}
\frac{1}{2} \sigma^{2} t^{2}|\operatorname{Im} \gamma(t)| & =|\operatorname{Im} \log f(t)| \\
& =|\operatorname{Im} f(t)|+O\left(t^{4}\right),
\end{aligned}
$$

$$
\frac{1}{2} \sigma^{2} \int_{0}^{A} \frac{|\operatorname{Im} \gamma(t)|}{t^{1+\delta}} d t=\int_{0}^{A} \frac{\left|\int_{-\infty}^{\infty}(\sin t u-t u) d F(u)\right|}{t^{3+\delta}} d t+O\left(A^{2-\delta}\right)
$$

and the finiteness of the integral on the right hand side under the condition $E\left|X_{i}\right|^{2+\delta}<\infty, 0 \leqq \delta<1$, follows from Lemma 5 of Heyde [4]. This result, together with (15), gives us (14) and thus completes the proof.

Addendum. Since the preparation of this paper there has appeared a related work "Convergence rates for the central limit theorem" by Friedman, Katz and Koopmans, Proc. nat. Acad. Sci. USA 56, 1062-1065 (1966). In this paper the convergence problem dealt with relates to a fixed $x$ and not to the maximum over all $x,-\infty<x<\infty$, as in the present work. In so far as the results are connected, those of the present paper are superior. Different methods are employed in the two papers.

\section{References}

1. BAUM, L. E., and M. L. KATZ: On the influence of moments on the asymptotic distribution of sums of random variables. Ann. math. Statistics 34, 1042-1044 (1963).

2. BiKJALis, A.: An estimate for the remainder term in the central limit theorem (Russian, English summary). Litovsk. Mat. Sbornik 4, 303-308 (1964).

3. Feller, W.: An introduction to probability theory and its applications, Vol. II. New York: Wiley 1966.

4. HEyde, C. C.: Some results on small deviation probability convergence rates for sums of independent random variables. Canadian J. Math. 18, 656-665 (1966).

5. Ibragimov, I. A.: The rate of convergence to the normal distribution. Soviet Math. Doklady 6, 580-582 (1965).

6. Katz, M. L.: Note on the Berry-Esseen theorem. Ann. math. Statistics 34, 1107-1108 (1963).

7. Linnik, Yu. V., and I. A. Ibragimov: Independent and stationary dependent random variables (Russian). Moscow 1965.

Department of Probability and Statistics

University of Sheffield, England, and

Matematisk Institut, Aarhus Universitet, Denmark 